ELECTRONIC LETTER

\title{
$M D R 1$, the blood-brain barrier transporter, is associated with Parkinson's disease in ethnic Chinese
}

\author{
C G L Lee, K Tang, Y B Cheung, L P Wong, C Tan, H Shen, Y Zhao, R Pavanni, E J D Lee, \\ M-C Wong, S S Chong, E K Tan
}

J Med Genet 2004;41:e60 (http://www.jmedgenet.com/cgi/content/full/41/5/e60). doi: 10.1136/jmg.2003.013003

$\mathrm{P}$ arkinson's disease is the second most common neurodegenerative disease after Alzheimer's disease. It is characterised by bradykinesia, rigidity, resting tremor, and postural instability. ${ }^{1}$ It is a genetically heterogeneous disorder. Pathogenic mutations in several genes-including $\alpha$-synuclein, Parkin, UCH-Ll (ubiquitin-C terminal hydrolaseLl) and $D J$-l-have previously been identified in rare monogenic forms of this disease showing autosomal dominant, autosomal recessive, or maternal transmission, with or without genetic anticipation. ${ }^{2} 3$ The more common, sporadic form of Parkinson's disease appears to result from an interaction between genetic and environmental factors. ${ }^{4}$ Polymorphisms in several genes, including those implicated in familial forms of the disease such as $\alpha$-synuclein ${ }^{5}$ and Parkin, ${ }^{67}$ are also reported to be associated with the sporadic form. ${ }^{8}$

Genetic susceptibility to sporadic Parkinson's disease was also found to be modulated by genes involved in xenobiotic management. A meta-analysis of 84 association studies of 14 genes showed that polymorphisms in four genes are significantly associated with the disease. ${ }^{9}$ These genes are either responsible for xenobiotic metabolism, such as $N A T 2^{10}{ }^{11}$ and GSTT1, ${ }^{12}$ or may interact with environmental agents, such as monoamine oxidase $(M A O B) .{ }^{13}$ Poor metaboliser alleles of the cytochrome $\mathrm{P} 450$ xenobiotic metabolism enzyme, CYP2D6, may also be associated with increased risk of Parkinson's disease. ${ }^{14-20}$ Furthermore, there may be sex effects in the association of CYP2D6 mutant alleles with Parkinson's disease. ${ }^{21}$

These genetic association studies corroborate epidemiological studies, which have long suggested that Parkinson's disease is associated with exposure to certain environmental xenobiotics. Although most of the specific agents remain to be identified, rural living, well water consumption, industrialisation, and herbicide/pesticide exposure have been implicated as potential risk factors. ${ }^{122} 23$

Another category of genes that may influence susceptibility to Parkinson's disease is the ATP binding cassette (ABC) superfamily of transporter genes which regulate the bioavailability of xenobiotics within critical tissues and cells in the body, of which the MDRl multidrug transporter or Pglycoprotein is the best characterised member. Unlike drug metabolising enzymes, whose major drug metabolising functions occur in the liver, the MDRl transporter is expressed at the interface of major organs. This pattern of distribution suggests that the $M D R 1$ transporter regulates the traffic of drugs and xenobiotics in the body at two levels: its expression in the epithelial cells of the gut serves as a first initial barrier regulating the absorption of xenobiotics into the body, while its expression at the blood-brain and bloodgerm cell/fetal interface serves as a second barrier controlling the uptake of xenobiotics into these sensitive tissues. ${ }^{24}$

The importance of the MDRl transporter as a component of the blood-brain barrier is evident in knockout mouse

\section{Key points}

- Seven single nucleotide polymorphisms (SNPs) spanning $\sim 100 \mathrm{~kb}$ of the MDR1 gene were examined in 206 Chinese patients with Parkinson's disease and 224 matched normal controls.

- Three SNPs-e12/1236(C/T), e21/2677(G/T/A), and e26/3435(C/T)-showed a significant association with Parkinson's disease. In particular, e12/ 1236T, e21/2677T, and e26/3435T, or haplotypes containing these alleles, were found to be overrepresented in the matched normal controls compared with the Parkinson patients.

- The significant effects of these SNPs were primarily observed in men and in patients with age of onset $\geqslant 60$ years; they were not associated with significant risk for Parkinson's disease in women or in patients with a younger age of onset ( $\leqslant 55$ years).

- It appears that the MDRI transporter is a significant modulator of susceptibility to Parkinson's disease among male ethnic Chinese $\geqslant 60$ years of age.

studies. Mdrla(-/-) mice were found to accumulate toxic levels of the anticancer drug, vinblastine, in the brain. ${ }^{25}$ Also, loperamide-an antidiarrhoeal narcotic analogue that normally does not enter the central nervous system (CNS) - was found to enter the brain of $m d r l a(-/-)$ mice, causing them to develop abnormal behaviour characteristic of toxicity to CNS permeable opiates (for example morphine). ${ }^{26}$ Hence, we hypothesised that functional polymorphisms in the MDRl gene may compromise its blood-brain barrier transporter function, increase accessibility of neurotoxic xenobiotics to the brain, and result in increased susceptibility to Parkinson's disease.

Several single nucleotide polymorphisms ( SNPs) have been identified in the MDRl gene, of which two (e21/2677(G/T/A) and e26/3435(C/T) ) have been reported to be associated with differences in MDRl expression and function, although the functional significance remains unclear. The non-synonymous SNP e21/2677(G/T/A) was reported to change the efflux of digoxin in cells in vitro in one study, ${ }^{27}$ but did not alter the efflux of several substrates in another study that used a different experimental system. ${ }^{28}$ The synonymous SNP e26/3435(C/T) has variously been associated with differences in MDRI protein expression and plasma drug concentration, ${ }^{27}{ }^{29-31}$ with drug induced side effects, ${ }^{32}$ and with drug response. $^{33}$ Recently, these two SNPs and a third one, el/ $-129(\mathrm{~T} / \mathrm{C})$, were examined in two case-control studies of approximately 100 patients with Parkinson's disease and 
matched normal controls. ${ }^{34}{ }^{35}$ No statistical significance was found between any of these SNPs and Parkinson's disease.

In this study, we examined seven SNPs as well as haplotypes of these SNPs spanning $\sim 100 \mathrm{~kb}$ in potentially functional regions of the $M D R l$ gene (that is, promoter region, coding regions, and 3'UTR) for an association with Parkinson's disease. We found a significant association between Parkinson's disease and the SNPs e12/1236(C/T), e21/2677(G/T/A), and e26/3435(C/T) (p values between 0.0367 and 0.00067$)$, or haplotypes of these SNPs $(p<0.05)$, in the Chinese population.

\section{METHODS}

\section{Study population}

All patients with Parkinson's disease and controls in this study were ethnic Chinese from Singapore. The Chinese in Singapore are predominantly descendents of migrants from south China. Individuals identified from the health screening programme in Singapore with no evidence of neurodegenerative disease on clinical examination were selected to serve as controls for the study. The diagnosis of Parkinson's disease was made by neurologists specialising in movement disorders according to the United Kingdom Parkinson's disease brain bank criteria. $^{36}$ DNA was isolated from blood samples collected from 206 patients with Parkinson's disease and 224 controls matched for age, sex, and ethnic group (table 1).

Ethical approval was obtained from the Singapore General Hospital research ethics committee.

\section{Genotyping}

The seven SNPs spanning $\sim 100 \mathrm{~kb}$ of the MDRl gene are located in five potentially functional genomic regions (promoter, exons 12, 21, 26, and 28) (fig 1). The five genomic segments were amplified in a single polymerase chain reaction, and all seven SNPs were genotyped by multiplex minisequencing as previously described. ${ }^{37}$

\section{Data analyses}

Genotype frequencies for the various SNPs in Parkinson's disease patients and controls were assessed for deviation from Hardy-Weinberg equilibrium using Pearson's $\chi^{2}$ test. $^{38}$ A log-linear model embedded within the EM algorithm was used to estimate haplotype frequencies and haplotypedisease association. ${ }^{39} 40$ The analyses assumed HardyWeinberg equilibrium but allowed for linkage disequilibrium. A likelihood ratio test was used to assess whether haplotypedisease association models fitted better than models assuming no haplotype-disease association. As the likelihood ratio test assessed models rather than particular haplotypes, we also estimated odds ratios (OR) for each haplotype to quantify the strength and direction of the association of individual haplotypes, using the more prevalent haplotypes as reference. We obtained $95 \%$ confidence intervals (CI) of the odds ratios by the profile likelihood approach; a 95\% CI that excluded the value of 1 indicated a significant relation between a particular haplotype and Parkinson's disease risk. $^{41}{ }^{42}$ The EM algorithm estimation was carried out using the Stata program.$^{41}$ All probability $(p)$ values were two sided, and a $\mathrm{p}$ value smaller than 0.05 was considered significant.

SNPs with frequencies below 5\% were excluded from the haplotype-disease association studies. In supplementary analyses, we examined the conditional independency of the excluded SNPs from Parkinson's disease given the flanking SNPs by a likelihood ratio test, ${ }^{39}$ to determine whether the inclusion of these SNPs could improve the haplotypeParkinson's disease (haplotype-PD) association models given the flanking SNPs.

In subset analyses we further explored whether the association of the various alleles/haplotypes in the MDR1 gene with Parkinson's disease differed between categories of sex and age of onset. As the average age of onset of Parkinson's disease is around 60 years (table 1), early onset was defined as developing the disease at or before the age of

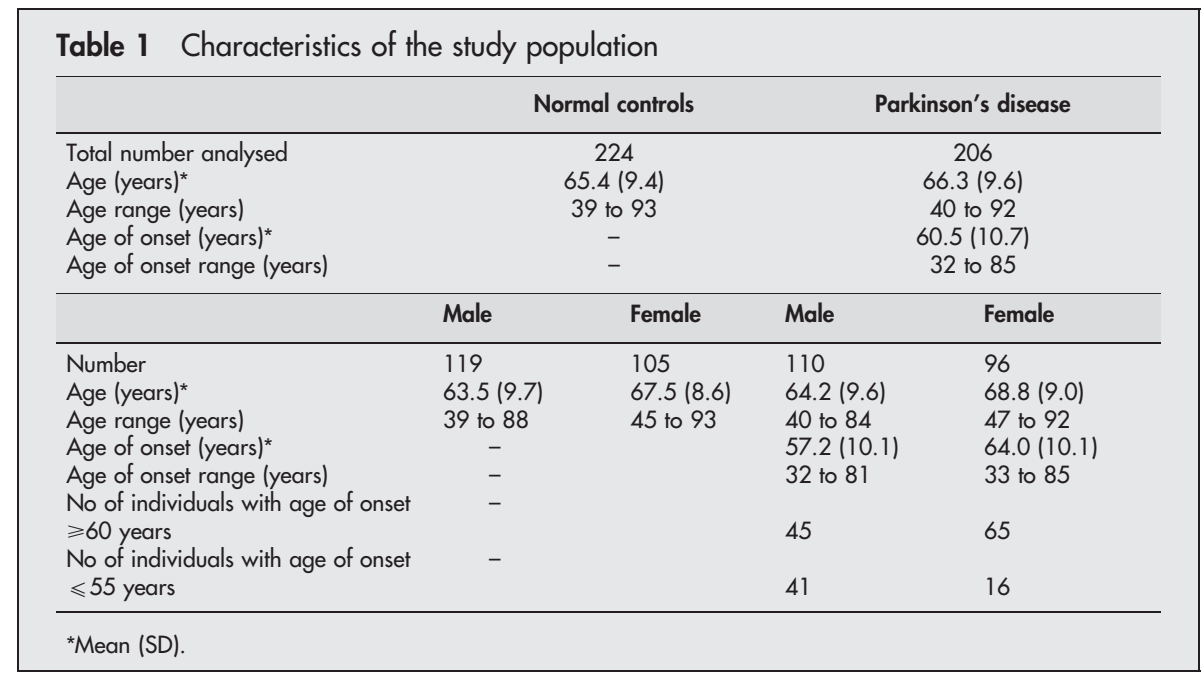

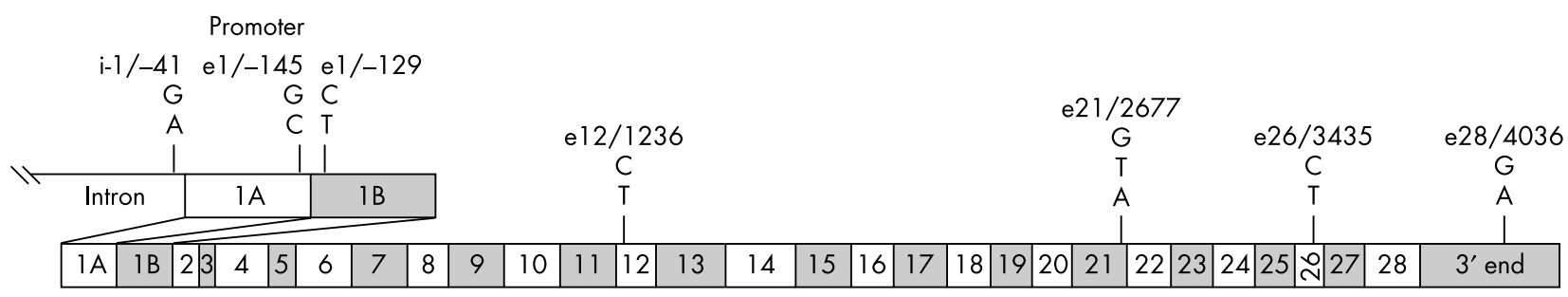

Figure 1 Schematic diagram showing relative positions of the SNP sites in the promoter and exons of the MDR1 gene. 
55, while late onset was defined as developing the disease at or after the age of 60. A gap of four years between 56 and 59 was not analysed, to allow for uncertainty in the ascertainment of the exact age of onset of some of the patients. Odds ratios and their confidence intervals were estimated separately in the different sex and age of onset groups. A sensitivity analysis was also carried out whereby we restricted the analysis of haplotype-disease association to subjects with phase-known haplotypes only. A logistic regression was used to estimate the odds ratio of disease.

\section{RESULTS}

As the genetic basis for complex disorders including Parkinson's disease is still unclear, there could be extensive allelic variation at any disease locus, resulting in multiple susceptibility alleles of independent origin present in the

Table 2 Association of single nucleotide polymorphisms (SNPs) or haplotypes of SNPs with Parkinson's disease

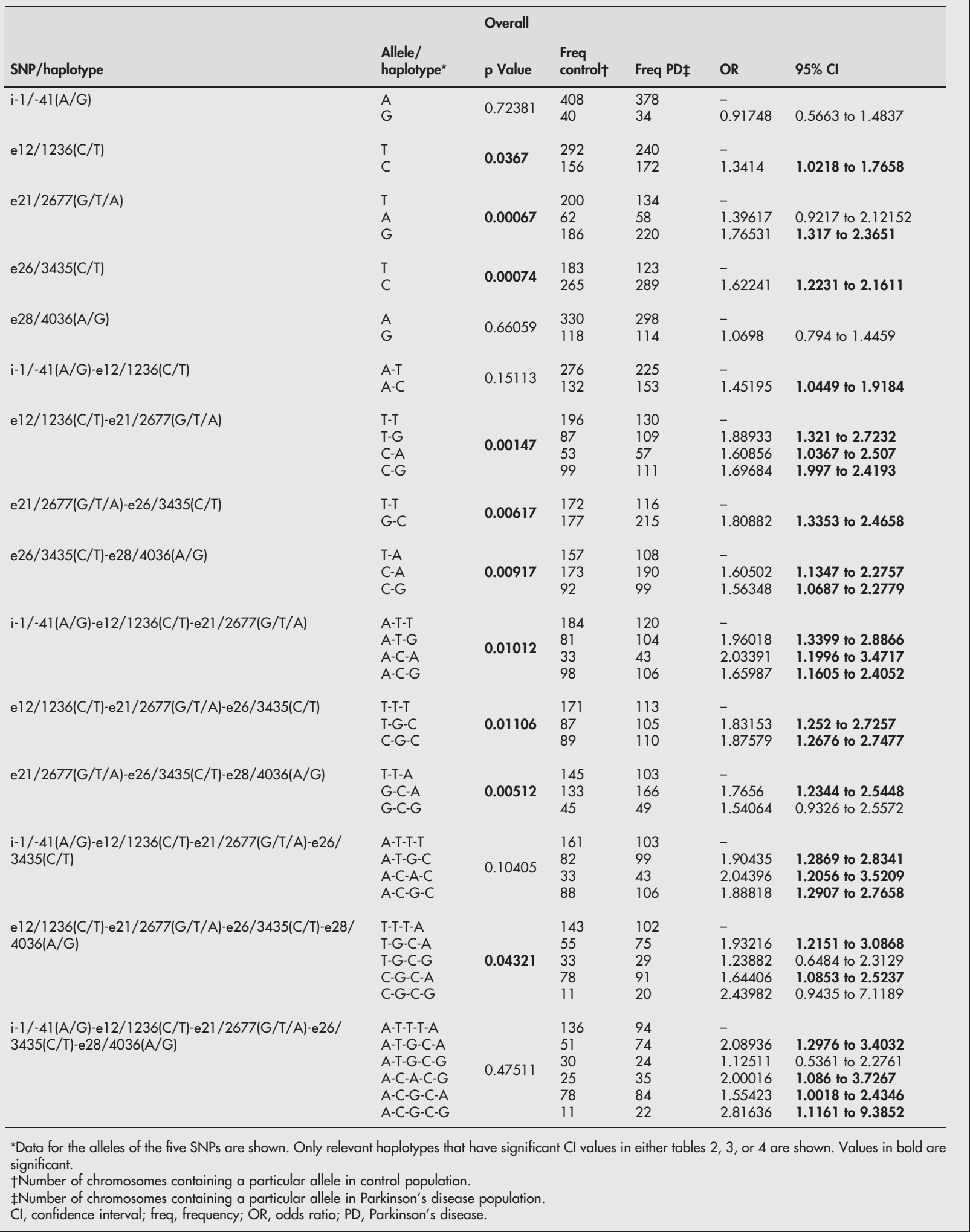




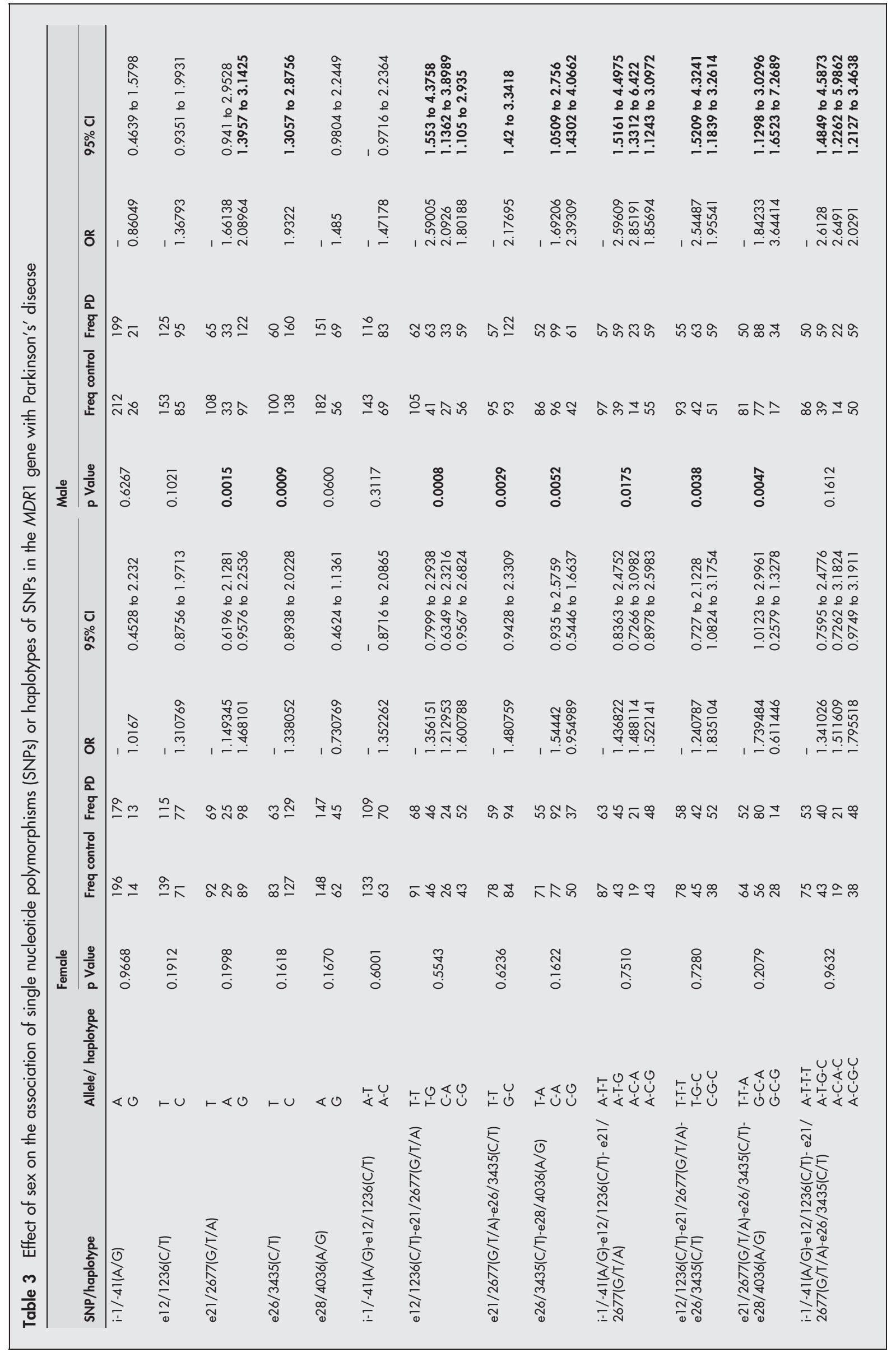




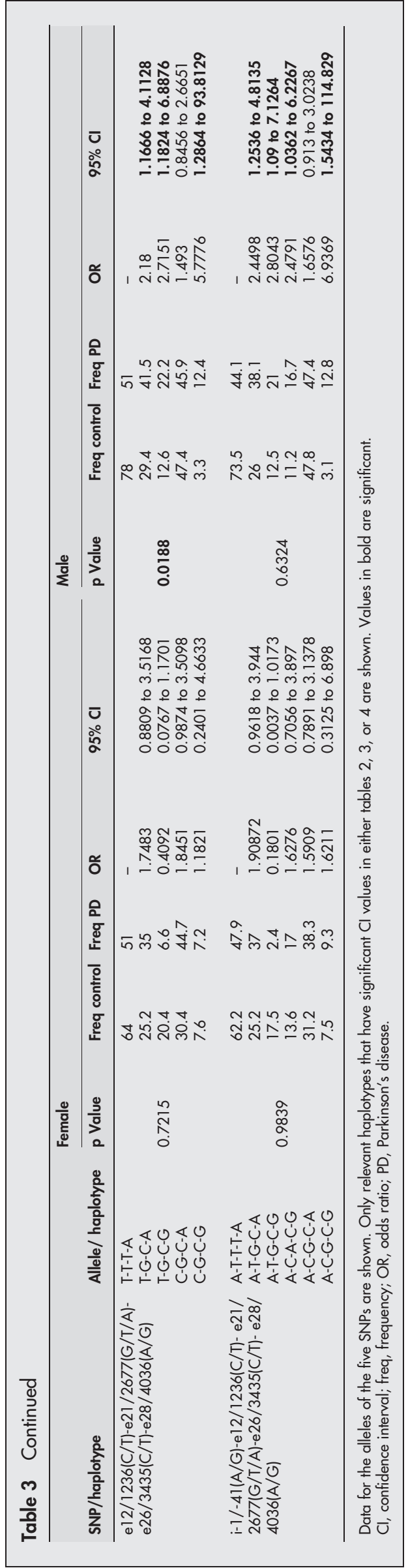

population. ${ }^{43-45}$ It has been suggested that analysis of haplotypes rather than individual SNPs may be more advantageous in the presence of multiple susceptibility alleles at a single disease locus. ${ }^{43}$ In this study, we examined the association of individual SNPs as well as SNP haplotypes with Parkinson's disease in ethnic Chinese.

Pearson's $\chi^{2}$ test showed that all seven SNPs in our study population were consistent with the Hardy-Weinberg equilibrium assumption (each $\mathrm{p}>0.05$ ).

\section{Association of MDR1 SNPs and their haplotypes with Parkinson's disease}

As shown in table 2, the C allele of SNP el2/1236(C/T) (OR $1.341(95 \% \mathrm{CI}, 1.022$ to 1.766$) ; \mathrm{p}=0.0367)$, the $\mathrm{G}$ allele of SNP e21/2677(G/T/A) (OR 1.765 (1.317 to 2.365); $\mathrm{p}=0.00067)$, and the $\mathrm{C}$ allele of SNP e26/3435(T/C) (OR $1.622(1.223$ to 2.161$) ; \mathrm{p}=0.00074)$ were individually significantly associated with a higher risk of developing Parkinson's disease. These three SNPs have previously been shown to be in tight linkage disequilibrium in the Chinese population. ${ }^{46}$ Calculated $\mathrm{p}$ values for all the possible haplotypes containing the above SNPs showed significant associations between these SNP combinations and Parkinson's disease $(p=0.04321$ to 0.00147$)$, except for three combinations containing SNP i-l/-4l(A/G) (i-l/-4l(A/ G)-el2/1236(C/T $) \quad(p=0.1511), \quad i-1 /-41(A / G)-e l 2 / 1236(C / T)$ -e21/2677(G/T/A)-e26/3435(C/T) $(\mathrm{p}=0.1041)$, and i-1/-4l (A/ G) -e12/1236(C/T)-e21/2677(G/T/A)-e26/3435(C/T)-e28/4036(A/ $\mathrm{G})(\mathrm{p}=0.4751)$ ) (table 2$)$. Even so, some specific haplotypes within these three SNP combinations were individually found to be associated with an increased risk of Parkinson's disease (table 2).

SNPs el/-145(C/G) and el/-129(T/C) were excluded from the haplotype-association analyses as the minor alleles of these SNPs occur at less than 5\% frequency. To evaluate whether the inclusion of these two SNPs would improve the haplotype-PD association models, we undertook conditional independence tests of the two SNPs from Parkinson's disease, given the flanking SNPs by the likelihood ratio test. It was found that these two SNPs did not improve the haplotype-PD association model significantly (each $\mathrm{p}>0.05$ ).

A sensitivity test using only phase-known haplotypes yielded similar results as EM estimated haplotype frequencies (data not shown), suggesting that the EM estimated haplotype frequencies were reliable.

\section{Sex differences in risk determination}

The characteristics of male and female Parkinson's disease patients in our study population were found to be different. The women tended to be older and to have a later age of disease onset than the men $(\mathrm{p}<0.05)$ (table 1$)$. We proceeded to examine whether there are sex specific associations between SNPs/haplotypes of the MDRl gene and Parkinson's disease. Our results showed that only haplotypes e12/1236C-e21/2677G-e26/3435C (OR 1.835 (95\% CI, 1.082 to 3.175 ) and e2l/2677G-e26/3435C-e28/4036A (OR 1.739 (1.012 to 2.996) were significantly associated with Parkinson's disease in women (table 3). In contrast, most of the MDRI SNPs and haplotypes that were significant in table 2 were also significant in men (table 3). Only SNP e12/ 1236C, and haplotypes i-1/-41A-e12/1236C, el2/1236C-e21/ 2677G-e26/3435C-e28/4036A, and i-1/-4lA-el2/1236C-e2l/ 2677G-e26/3435C-e28/4036A were not significantly associated with Parkinson's disease in men, although their association with the disease in the overall population was significant. In addition, haplotypes e21/2677G-e26/3435C-e28/ 4036G (OR 3.644 ( 1.652 to 7.269 ), el2/1236T-e2l/2677Ge26/3435C-e28/4036G (OR 2.715 ( 1.182 to 6.888), el2/ 1236C-e2l/2677G-e26/3435C-e28/4036G (OR 5.778 (1.286 to 


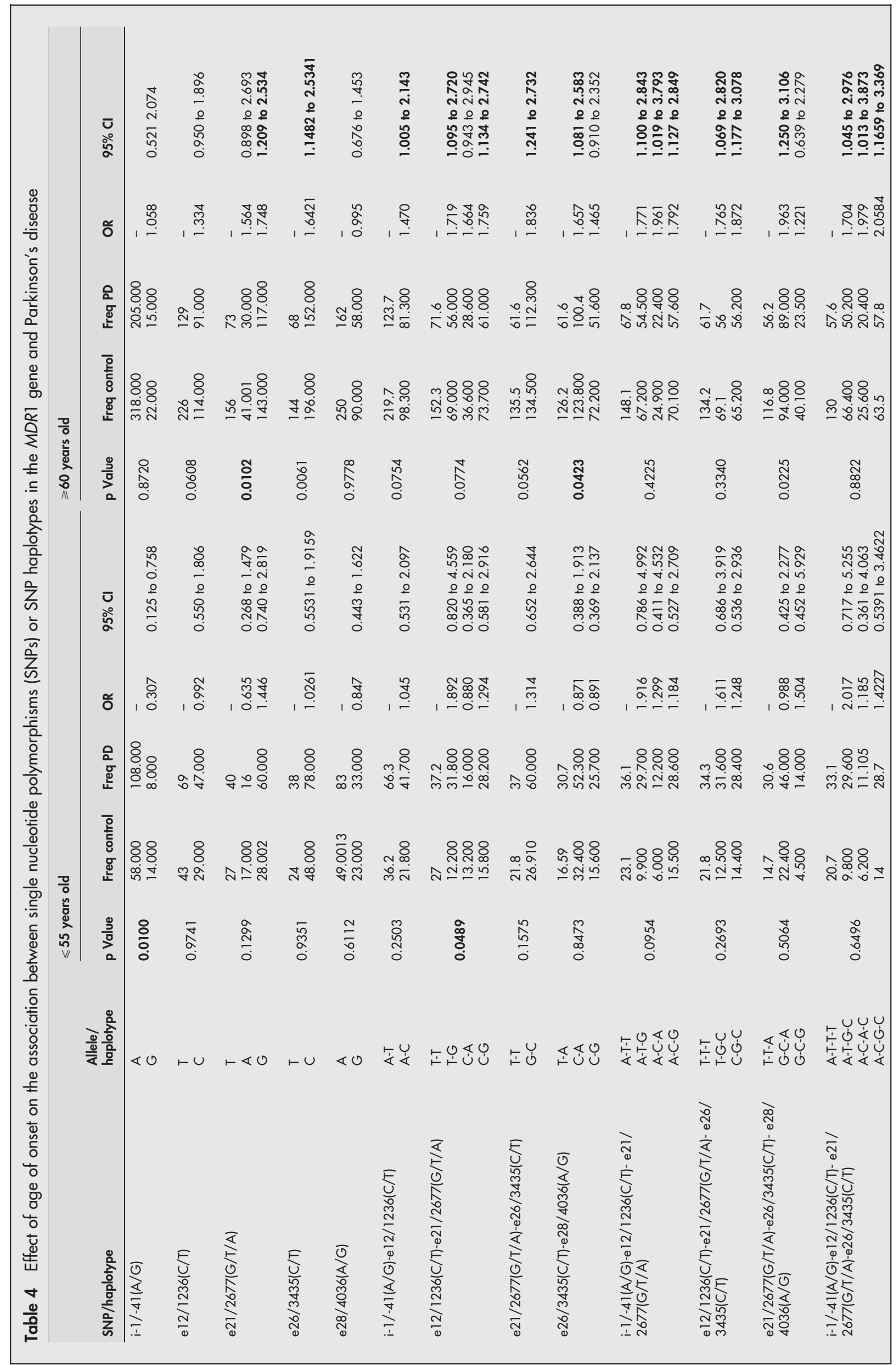




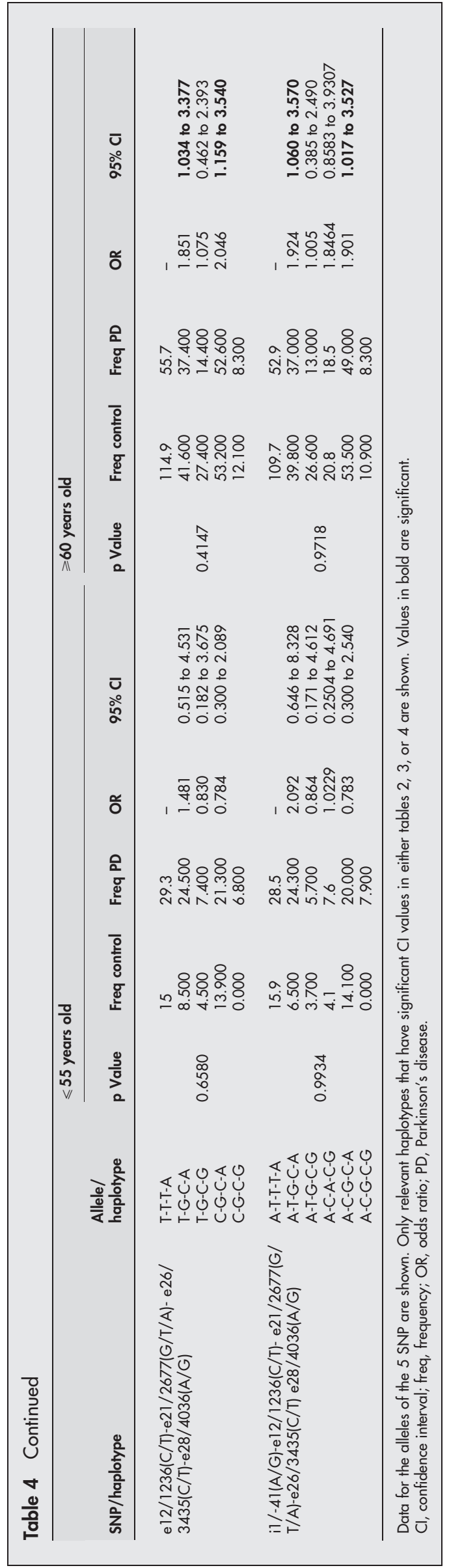

93.813), and i-1/-41A-el2/1236T-e21/2677G-e26/3435C-e28/ 4036G (OR 2.804 (1.090 to 7.126) were significantly associated with Parkinson's disease in men but not overall (table 3).

\section{Role of SNPs/haplotypes in the MDR1 gene in later onset of Parkinson's disease}

Interesting observations were made when we examined the age of onset specific association of SNPs/haplotypes in the MDRl gene with Parkinson's disease. While the promoter SNP i-l/-4l(A/G) was found not to be associated with Parkinson's disease in our overall or sex specific analyses, the low frequency $G$ allele of this SNP was found to be significantly associated $(p=0.01)$, with a decreased risk of developing Parkinson's disease at or before the age of 55 years (OR 0.307 (95\% CI, 0.125 to 0.758$)$ (table 4). Conversely, SNPs e2 l/2677(G/T/A) $(p=0.0102), e 26 / 3435(\mathrm{C} /$ T) $(\mathrm{p}=0.0061)$, and SNP combinations e26/3435(C/T)-e28/ 4036(A/G) $(\mathrm{p}=0.0423)$ and e2 l/2677(G/T/A)-e26/3435(C/T)e28/4036(A/G) $(\mathrm{p}=0.0225)$ were associated with increased risk of developing Parkinson's disease at or after age 60, with SNPs e2 l/2677G (OR 1.748 (1.209 to 2.534)) and e26/3435C (OR 1.642 ( 1.148 to 2.354)), and haplotypes e26/3435C-e28/ 4036A (OR 1.657 (1.081 to 2.583)) and e2l/2677G-e26/ 3435C-e28/4036A (OR 1.963 (1.250 to 3.106)) being associated with the increased risk (table 4). Some haplotypes that include either or both of the SNPs e21/2677(G/T/A) and e26/ $3435(\mathrm{C} / \mathrm{T})$ were also associated with an increased risk of developing Parkinson's disease (table 4). Curiously, although SNPs il/-4l(A/G) and el2/1236(C/T) were not individual risk factors, the haplotype i-1/-41A-el2/1236C (OR 1.470 (1.005 to 2.143$)$ ) was significantly associated with increased risk of late onset Parkinson's disease (table 4).

Overall, the results from table 4 suggest that SNP i-1/ $-41(\mathrm{~A} / \mathrm{G})$ may be associated with decreased risk for developing Parkinson's disease at or before the age of 55, while SNPs e21/2677(G/T/A) and e26/3435(C/T) and haplotypes containing these SNPs are associated with later onset disease $(\geqslant 60$ years).

\section{DISCUSSION}

Environmental xenobiotics have been implicated in the development of Parkinson's disease, a complex genetically heterogeneous disorder. ${ }^{122}{ }^{23}$ The blood-brain barrier plays an important role in regulating the traffic of environmental xenobiotics in the brain, and individual differences in the "quality" of this barrier may influence the susceptibility to Parkinson's disease. The MDRl multidrug transporter represents an important component of the blood-brain barrier and has been shown to regulate the uptake of drugs and xenobiotics into this sensitive organ..$^{26}{ }^{26}$ It is conceivable that polymorphisms which alter the expression levels or transport ability of this transporter could result in altered susceptibility to neurotoxic substances and thus alter the genetic threshold for the development of Parkinson's disease.

Two recent case-control studies have examined the role of MDRl gene polymorphisms (SNPs el/-129(T/C), e21/2677(G/ $\mathrm{T} / \mathrm{A})$, and $\mathrm{e} 26 / 3435(\mathrm{C} / \mathrm{T}))$ in Parkinson's disease development. The studies involved approximately 100 white Italian and Polish patients and 100 controls from the same geographical regions. ${ }^{34}{ }^{35}$ No significant associations between these SNPs and Parkinson's disease were detected. However, our present study of 206 Chinese patients and 224 controls showed that three SNPs-e12/1236(C/T) $(p=0.0367)$, e2l/ $2677(\mathrm{G} / \mathrm{T} / \mathrm{A})(\mathrm{p}=0.00067)$, and $\mathrm{e} 26 / 3435(\mathrm{C} / \mathrm{T})(\mathrm{p}=0.00074)$, all in tight linkage disequilibrium with each other ${ }^{46}$-are significantly associated with an altered risk of developing Parkinson's disease (table 2). The odds ratios of the haplotypes that were associated with Parkinson's disease 
were not very high. These observations are, however, consistent with the widely held view that Parkinson's disease is a complex disorder involving the interaction of multiple genes with different environmental factors, whereby the individual contribution of each causative gene may not be large.

We recently found strong evidence of positive selection for the e21/2677T and e26/3435T alleles in the Chinese, but only marginal evidence for this in white Americans (Tang K, Wong L, Lee E, et al, Human Molecular Genetics (in press)). The Chinese samples in that study were from anonymised umbilical cord blood from Chinese neonates, and allele frequencies of the seven SNPs were found to be very similar to those in the present study. When we used cord blood DNA samples as controls to compare against the Parkinson's disease samples, we obtained a similar, statistically significant association between Parkinson's disease and these two SNPs (data not shown). The strong evidence of a recent positive selection for the T alleles of these two SNPs supports our current observation that these alleles are significantly underrepresented in patients with Parkinson's disease compared with unaffected controls, suggesting that the $\mathrm{T}$ alleles of these SNPs may confer better protection for the brain against xenobiotic insults in the Chinese population.

It is possible that the earlier Italian and Polish association studies did not detect a significant statistical association because of their limited sample size. There may be another reason why neither study was able to detect a significant association between any MDRl SNPs and Parkinson's disease. If we assume that the Italian and Polish subjects ${ }^{34} 35$ were genetically similar to white Americans, their MDR 1 haplotype and LD profiles may not favour the detection of associations. Our observation of only marginal evidence of recent positive selection in white Americans compared with the Chinese supports this hypothesis. Nonetheless, it remains to be determined whether the white Italians and Poles are in fact similar to white Americans in their underlying genetic architecture at this locus.

It is possible that either SNP e2 $1 / 2677(\mathrm{G} / \mathrm{T} / \mathrm{A})$ or e26/ $3435(\mathrm{C} / \mathrm{T})$ could be potential causal SNPs as they had much lower $\mathrm{p}$ values than SNP e12/1236(C/T). Consistent with our observation that individuals carrying the $\mathrm{G}$ allele at the nonsynonymous SNP e21/2677(G/T/A) have a higher risk of developing Parkinson's disease, the MDRl transporter carrying the e2l/2677G allele-coding for Ala at amino acid position 893-has been shown to be a less effective transporter than one carrying the $\mathrm{T}$ allele (Ser 893). ${ }^{27}$ The synonymous SNP e26/3435(C/T) appears to be associated with altered MDRl transporter expression and function. While several reports found that the $\mathrm{T}$ allele is associated with lower MDRl expression, ${ }^{29} 303348$ resulting in lower efflux or higher plasma levels of drugs and xenobiotics, ${ }^{29} 30$ others have reported lower drug plasma concentration in individuals carrying the $\mathrm{T}$ allele. ${ }^{27}{ }^{313}$ Most of these studies examined only SNP e26/3435(C/T) without taking into account the underlying haplotype and linkage disequilibrium architecture of the study population. Detailed characterisation of the genetic and evolutionary history of the entire $M D R 1$ gene in each study population, and the influence of recent events in the history of each population on linkage disequilibrium and the likelihood of detecting an association, could resolve these conflicting reports. Our data showing an association between e26/3435T and a lower risk of developing Parkinson's disease support observations that the T allele alters MDRl function, resulting in a greater efflux of drugs or xenobiotics. Although SNP e26/3435(C/T) is a synonymous SNP and does not result in an amino acid change, there are several possible explanations for this observation. The observed correlation with e26/3435T could reflect either differential codon usage of the $\mathrm{C}$ or $\mathrm{T}$ allele at the wobble position of the isoleucine codon, or allele specific differences in RNA folding, ${ }^{49}$ sometimes influencing RNA processing ${ }^{50}$ or splicing, ${ }^{51}{ }^{52}$ or differences in translation control ${ }^{53}$ and regulation. ${ }^{54}$ It is also possible that neither SNP e21/2677(G/T/A) nor e26/3435(C/T) represents the causal SNP, but that they are merely in strong linkage disequilibrium with an unobserved causal SNP. A strong association of these two SNPs with Parkinson's disease could suggest that the linked causal variant resides within a region defined by strong LD.

An interesting observation was made when male and female patients with Parkinson's disease were investigated independently-the MDRl gene appears to play a more important role in determining risk of developing the disease in men than in women (table 3). This is consistent with the view that the MDRI transporter regulates the accumulation of neurotoxic xenobiotics in the brain to modulate the risk of developing Parkinson's disease. As older women in urban Singapore are primarily home makers while men often work out of doors, it is conceivable that the observed greater risk for Parkinson's disease in men compared with women is related to increased exposure to environmental susceptibility factors among men, given the same genetic risk factors in the two sexes.

When patients with Parkinson's disease were compared on the basis of their age at disease onset, we found that several polymorphisms in the MDRl gene seemed to play a greater role in later onset disease ( $\geqslant 60$ years) (table 4$)$. One hypothesis is that, in individuals with particular $M D R \mathrm{l}$ genotypes (for example, el2/1236C, e21/2677G, e26/3435C) and haplotypes, the blood-brain barrier allows neurotoxic xenobiotics easier access and gradual accumulation in the brain, eventually leading to Parkinson's disease. Conversely, individuals with the alternative alleles (that is, el2/1236T, e21/2677T and e26/3435T) are better protected from xenobiotic insults and hence from Parkinson's disease. In contrast, early onset Parkinson's disease is probably a result of other genetic factors and hence is less dependent on genetic variation at the $M D R 1$ locus.

The promoter SNP i-1/-4l(A/G), which resides in a putative CCAAT box, was found to influence the risk of Parkinson's disease in patients with a younger age of onset $(p=0.01)$ (table 4). The G allele of this SNP appeared to protect individuals from Parkinson's disease (OR 0.307 (95\% CI, 0.125 to 0.758$)$ ). This observation, however, should be interpreted cautiously, given the low frequency $(<10 \%)$ of $\mathrm{i}-\mathrm{l} /-4 \mathrm{lG}$ in the general population and the resultant sample sizes in this comparison.

\section{Conclusions}

We have produced strong statistical evidence that particular alleles and haplotypes of MDRl SNPs-e12/1236(C/T), e2l/ 2677(G/T/A), and e26/3435(C/T) - are important risk factors for the development of Parkinson's disease in ethnic Chinese, especially in men, through sex associated lifestyle differences, and in individuals with a later age of onset ( $\geqslant 60$ years). The wide variations in allele frequencies of the MDR 1 SNPs (especially SNP el2/1236(C/T), e21/2677(G/T/A), and e26/ $3435(\mathrm{C} / \mathrm{T})$ ) among different ethnic populations ${ }^{46}$ may account for the differences in the ability to detect an association between MDRl and Parkinson's disease in other ethnic groups, especially if the increase in relative risk is small.

\section{ACKNOWLEDGEMENTS}

This study was supported by a grant from the National Medical Research Council, Singapore (NMRC/0657/2002) to CGL, SSC, and EJDL. 


\section{Authors' affiliations}

C G L Lee, K Tang, L P Wong, Departments of Biochemistry, National University of Singapore, Singapore

Y B Cheung, Biostatistics Unit, Division of Clinical Trials and Epidemiological Sciences, National Cancer Centre, Singapore C Tan, H Shen, Y Zhao, E K Tan, Department of Neurology, Singapore General Hospital

R Pavanni, M-C Wong, National Neuroscience Institute, Singapore

E J D Lee, Departments of Pharmacology, National University of Singapore

S S Chong, Departments of Paediatrics, National University of Singapore Conflicts of interest: none declared

Correspondence to: Dr Caroline G Lee, Division of Medical Sciences, National Cancer Centre, Level 6, Lab 5, 11 Hospital Drive, Singapore 169610, Singapore; bchleec@nus.edu.sg

\section{REFERENCES}

1 Langston JW. Epidemiology versus genetics in Parkinson's disease: progress in resolving an age-old debate. Ann Neurol 1998;44(suppl 1):S45-52

2 Mouradian MM. Recent advances in the genetics and pathogenesis of Parkinson disease. Neurology 2002:58:179-85

3 Bonifati V, Rizzu P, van Baren MJ, et al. Mutations in the DJ-1 gene associated with autosomal recessive early-onset parkinsonism. Science 2003;299:256-9.

4 Maimone D, Dominici R, Grimaldi LM. Pharmacogenomics of neurodegenerative diseases. Eur J Pharmacol 2001;413:11-29.

5 Farrer M, Maraganore DM, Lockhart $P$, et al. alpha-Synuclein gene haplotypes are associated with Parkinson's disease. Hum Mol Genet 2001;10:1847-51.

6 Wang $M$, Hattori N, Matsumine H, et al. Polymorphism in the parkin gene in sporadic Parkinson's disease. Ann Neurol 1999:45:655-8.

7 Satoh J, Kuroda Y. Association of codon 167 Ser/Asn heterozygosity in the parkin gene with sporadic Parkinson's disease. Neuroreport 1999; 10:2735-9.

8 de Silva HR, Khan NL, Wood NW. The genetics of Parkinson's disease. Curr Opin Genet Dev 2000;10:292-8.

9 Tan EK, Khajavi M, Thornby Jl, et al. Variability and validity of polymorphism association studies in Parkinson's disease. Neurology 2000;55:533-8.

10 Bandmann O, Vaughan JR, Holmans $\mathrm{P}$, et al. Detailed genotyping demonstrates association between the slow acetylator genotype for $\mathrm{N}$ acetyltransferase 2 (NAT2) and familial Parkinson's disease. Mov Disord 2000;15:30-5

11 Harhangi BS, Oostra BA, Heutink P, et al. N-acetyltransferase-2 polymorphism in Parkinson's disease: the Rotterdam study. J Neurol Neurosurg Psychiatry 1999;67:518-20.

12 Stroombergen MC, Waring RH. Determination of glutathione S-transferase $\mathrm{mu}$ and theta polymorphisms in neurological disease. Hum Exp Toxicol 1999;18:141-5.

13 Checkoway H, Franklin GM, Costa-Mallen P, et al. A genetic polymorphism of MAO-B modifies the association of cigarette smoking and Parkinson's disease. Neurology 1998;50:1458-61

14 Armstrong M, Daly AK, Cholerton S, et al. Mutant debrisoquine hydroxylation genes in Parkinson's disease. Lancet 1992:339:1017-18.

15 Smith CA, Gough AC, Leigh PN, et al. Debrisoquine hydroxylase gene polymorphism and susceptibility to Parkinson's disease. Lancet 1992;339:1375-7.

16 Rostami-Hodjegan A, Lennard MS, Woods HF, et al. Meta-analysis of studies of the CYP2D6 polymorphism in relation to lung cancer and Parkinson's disease. Pharmacogenetics 1998;8:227-38.

17 McCann SJ, Pond SM, James KM, et al. The association between polymorphisms in the cytochrome P-450 2D6 gene and Parkinson's disease: a case-control study and meta-analysis. J Neurol Sci 1997;153:50-3.

18 Christensen PM, Gotzsche PC, Brosen K. The sparteine/debrisoquine (CYP2D6) oxidation polymorphism and the risk of Parkinson's disease: a meta-analysis. Pharmacogenetics 1998;8:473-9.

19 Riedl AG, Watts PM, Jenner P, et al. P450 enzymes and Parkinson's disease: the story so far. Mov Disord 1998;13:212-20.

20 Landi MT, Ceroni M, Martignoni E, et al. Gene-environment interaction in parkinson's disease. The case of CYP2D6 gene polymorphism. Adv Neurol 1996;69:61-72.

21 Gerard N, Panserat S, Lucotte G. Roles of gender, age at onset and environmental risk in the frequency of CYP2D6-deficient alleles in patients with Parkinson's disease. Eur Neurol 2002;48:114-15.

22 Bonnet AM, Houeto JL. Pathophysiology of Parkinson's disease. Biomed Pharmacother 1999;53:117-21.

23 Koller W, Vetere-Overfield B, Gray C, et al. Environmental risk factors in Parkinson's disease. Neurology 1990:40:1218-21.

24 Lee CG, Gottesman MM. HIV-1 protease inhibitors and the MDR1 multidrug transporter [editorial]. J Clin Invest 1998;101:287-8.
25 Schinkel AH, Smit J, van Tellingen $O$, et al. Disruption of the mouse mdr la Pglycoprotein gene leads to a deficiency in the blood-brain barrier and to increased sensitivity to drugs. Cell 1994;77:491-502.

26 Schinkel AH, Wagenaar E, Mol CA, et al. P-glycoprotein in the blood-brain barrier of mice influences the brain penetration and pharmacological activity of many drugs. J Clin Invest 1996;97:2517-24.

27 Kim RB, Leake BF, Choo EF, et al. Identification of functionally variant MDR 1 alleles among European Americans and African Americans. Clin Pharmacol Ther 2001;70:189-99.

28 Kimchi-Sarfaty C, Gribar JJ, Gottesman MM. Functional characterization of coding polymorphisms in the human MDR1 gene using a vaccinia virus expression system. Mol Pharmacol 2002;62(1):1-6.

29 Hoffmeyer S, Burk O, von Richter O, et al. Functional polymorphisms of the human multidrug-resistance gene: multiple sequence variations and correlation of one allele with P-glycoprotein expression and activity in vivo. Proc Natl Acad Sci USA 2000;97:3473-8.

30 Hitzl M, Drescher S, van der Kuip H, et al. The C3435T mutation in the human MDR1 gene is associated with altered efflux of the P-glycoprotein substrate rhodamine 123 from CD56+ natural killer cells. Pharmacogenetics 2001;11:293-8.

31 Sakaeda T, Nakamura T, Horinouchi M, et al. MDR1 genotype-related pharmacokinetics of digoxin after single oral administration in healthy Japanese subjects. Pharm Res 2001;18:1400-4.

32 Roberts $R$, Joyce $\mathrm{P}$, Mulder R, et al. A common P-glycoprotein polymorphism is associated with nortriptyline-induced postural hypotension in patients treated with major depression. Pharmacogenomics 2002;2:191-6.

33 Fellay J, Marzolini C, Meadon E, et al. Response to antiretroviral treatment in HIV-1-infected individuals with allelic variants of the multidrug resistance transporter 1: a pharmacogenetic study. Lancet 2002;359:30-6.

34 Furuno T, Landi MT, Ceroni M et al. Expression polymorphism of the bloodbrain barrier component P-glycoprotein (MDR1) in relation to Parkinson's disease. Pharmacogenetics 2002;12:529-34.

35 Drozdzik M, Bialecka M, Mysliwiec K, et al. Polymorphism in the Pglycoprotein drug transporter MDR1 gene: a possible link between environmental and genetic factors in Parkinson's disease. Pharmacogenetics 2003;13:259-63.

36 Hughes AJ, Daniel SE, Kilford L, et al. Accuracy of clinical diagnosis of idiopathic Parkinson's disease: a clinico-pathological study of 100 cases. J Neurol Neurosurg Psychiatry 1992;55:181-4.

37 Gwee PC, Tang K, Chua JM, et al. Simultaneous genotyping of seven singlenucleotide polymorphisms in the MDR1 gene by single-tube multiplex minisequencing. Clin Chem 2003;49:672-6.

38 Sham P. Statistics in human genetics. London: Arnold, 1998.

39 Bitti PP, Murgia BS, Ticca A, et al. Association between the ancestral haplotype HLA A30B18DR3 and multiple sclerosis in central Sardinia. Genet Epidemiol 2001;20:271-83.

40 Chiano MN, Clayton DG. Fine genetic mapping using haplotype analysis and the missing data problem. Ann Hum Genet 1998;62:55-60.

41 Mander AP. Haplotype analysis in population-based association studies. The Stata Journal 2001;1:58-75.

42 McCullagh P, Nelder JA. Generalized linear models. London: Chapman and Hall, 1989.

43 Morris RW, Kaplan NL. On the advantage of haplotype analysis in the presence of multiple disease susceptibility alleles. Genet Epidemiol 2002;23:221-33.

44 Terwilliger JD, Weiss KM. Linkage disequilibrium mapping of complex disease: fantasy or reality? Curr Opin Biotechnol 1998;9:578-94.

45 Pritchard JK. Are rare variants responsible for susceptibility to complex diseases? Am J Hum Genet 2001;69:124-37.

46 Tang K, Ngoi SM, Gwee PC, et al. Distinct haplotype profiles and strong linkage disequilibrium at the MDR1 multidrug transporter gene locus in three ethnic Asian populations. Pharmacogenetics 2002;12:437-50.

47 Kim RB, Fromm MF, Wandel C, et al. The drug transporter P-glycoprotein limits oral absorption and brain entry of HIV-1 protease inhibitors. J Clin Invest 1998;101:289-94.

48 Tanabe M, leiri I, Nagata N, et al. Expression of P-glycoprotein in human placenta: relation to genetic polymorphism of the multidrug resistance (MDR)1 gene. J Pharmacol Exp Ther 2001;297:1137-43.

49 Shen LX, Basilion JP, Stanton VP. Single-nucleotide polymorphisms can cause different structural folds of mRNA. Proc Natl Acad Sci USA 1999;96:7871-6.

50 Allain FH, Gubser CC, Howe PW, et al. Specificity of ribonucleoprotein interaction determined by RNA folding during complex formulation. Nature 1996:380:646-50.

51 Coleman TP, Roesser JR. RNA secondary structure: an important cis-element in rat calcitonin/CGRP pre-messenger RNA splicing. Biochemistry 1998;37:15941-50.

52 Liu HX, Cartegni L, Zhang MQ, et al. A mechanism for exon skipping caused by nonsense or missense mutations in BRCAl and other genes. Nat Genet $2001 ; 27: 55-8$.

53 Shen LX, Tinoco I. The structure of an RNA pseudoknot that causes efficient frameshiffing in mouse mammary tumor virus. J Mol Biol 1995;247:963-78.

54 Addess KJ, Basilion JP, Klausner RD, et al. Structure and dynamics of the iron responsive element RNA: implications for binding of the RNA by iron regulatory binding proteins. J Mol Biol 1997;274:72-83 